\title{
FIBROSE AURICULAR SECUNDÁRIA A OTOHEMATOMA EM FELINO: TERAPÊUTICA COM GLICOCORTICÓIDE - RELATO DE CASO
}

\author{
TILLMANN, Mariana Teixeira; \\ MENDES, Claudia Beatriz De Mello; \\ FELIX, Anelize de Oliveira Campello ${ }^{3}$; \\ MUELLER, Eduardo Negri ${ }^{4}$; \\ NOBRE, Márcia de Oliveira ${ }^{5}$.

\footnotetext{
${ }^{1}$ Doutoranda, Programa de Pós-Graduação em Veterinária, UFPEL, ${ }^{2}$ Acadêmica da Faculdade de Veterinária da UFPEL, ${ }^{3}$ Médica Veterinária, Doutora, Biotério Central da Faculdade de Veterinária, UFPEL, ${ }^{4}$ Professor Doutor do Instituto Federal Catarinense-Campus Concórdia, ${ }^{5}$ Professora Doutora- Departamento de Clinicas Veterinária da Faculdade de Veterinária da UFPEL.
}

\section{RESUMO}

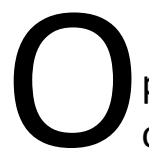

otohematoma se caracteriza pelo acúmulo sanguíneo na face convexa da orelha podendo desenvolver fibrose cicatricial quando não tratado adequadamente. 0 objetivo deste trabalho foi relatar o caso de um felino com presença de fibrose no pavilhão auditivo esquerdo, submetido à terapia com acetato de metilprednisolona infiltrativo. O paciente apresentava histórico de otohematoma não tratado cirurgicamente, presença de aumento de volume no pavilhão auricular esquerdo e leve inclinação da cabeça para o mesmo lado. A massa foi tratada por dois meses com $1 \mathrm{mg} / \mathrm{kg}$ de acetato de metilprednisolona intralesional em 2 doses com intervalos de 30 dias. 0 conduto auditivo do felino foi avaliado a cada 30 dias por otoscopia e a massa fibrótica foi mensurada com um paquímetro. $\mathrm{Na}$ inspeção da massa fibrótica, foi constatada redução gradativa da área de fibrose a cada avaliação $\left(200,140\right.$ e $137 \mathrm{~mm}^{2}$ ) sendo que na segunda avaliação o paciente apresentava a cabeça ereta. Dessa maneira, conclui-se que a aplicação por 2 meses de 1 $\mathrm{mg} / \mathrm{kg}$ de acetato de metilprednisolona infiltrativo em duas doses com intervalo de 30 dias entre as aplicações, apresentou resultado satisfatório para o tratamento de fibrose auricular decorrente de otohematoma.

Palavras-chave: Corticóide. Enfermidades. Gato. Ouvido. 


\section{INTRODUÇÃO}

Os otohematomas são afecções raras em gatos e comum em cães e para sua resolução o tratamento mais indicado é a correção cirúrgica. No entanto, prévio ao procedimento cirúrgico é necessária a investigação e a terapia corretiva da causa primária ao hematoma auricular para prevenir reincidências (EVANGELISTA et al., 2012; SCHIOCHET et al., 2010). Nos casos em que o tratamento adequado não é realizado, podem ocorrer recidivas e/ou fibrose cicatricial devido à formação de tecido conjuntivo para reparar o trauma na cartilagem do pavilhão auricular (HARVEY et al., 2004). Após a formação dessa fibrose tornase difícil a resolução do quadro clínico e, na literatura veterinária, é escassa a informação sobre esse assunto (SCHOSSLER et al., 2007).

Uma das causas predisponentes ao otohematoma é a otite que se caracteriza por ser uma inflamação do meato acústico e possuir etiologia multifatorial sendo classificada em externa, média e interna de acordo com sua a localização anatômica na orelha (NASCENTE et al., 2006). A otite externa é uma enfermidade com alta casuística na clínica de pequenos animais, com maior prevalência em caninos, já nos felinos a otite externa parasitária é mais freqüente, mas em geral as demais causas de otite externa são incomuns (COLE, 2004; SCHIOCHET et al., 2010). A inflamação da orelha externa ocasiona alterações no órgão, tais como mudança do $\mathrm{pH}$, da umidade, da temperatura e consequentemente da microbiota, o que facilita o desenvolvimento de agentes secundários e oportunistas (LOBELL et al., 1995). Devido ao prurido ocasionado pela enfermidade, os sinais clínicos mais frequentes são o sacudir da cabeça e o movimento oto-podal, sendo essas as principais causas do hematoma auricular. $\mathrm{O}$ ato de coçar a orelha está relacionado ao otohematoma pela possibilidade de trauma nos vasos sanguíneos da pina auricular, ocasionando um hematoma, e presença de fluído seroso na região entre a cartilagem da orelha externa e a pele (EVANGELISTA et al., 2012; HARVEY et al., 2004). Este trabalho tem como objetivo relatar o caso de um felino com presença de fibrose no pavilhão auditivo externo esquerdo, submetido à terapia com acetato de metilprednisolona infiltrativo ( Depo Medrol Pfizer ${ }^{\circledR}$ ). 


\section{RELATO DE CASO}

No Hospital de Clínicas Veterinária da Universidade Federal de Pelotas, foi atendido um felino, macho, da raça persa, pesando $4,3 \mathrm{~kg}$, de três anos de idade. A queixa principal do proprietário era a presença de uma leve inclinação da cabeça do gato para o lado esquerdo. No histórico do paciente foi relatado que esse teve uma otite externa e que, ao redor de um mês, apresentou remissão do quadro clínico. No período em que foi diagnosticada a otite, observou-se a presença de um hematoma auricular no pavilhão auditivo externo esquerdo, que foi puncionado com intuito de reduzir o otohematoma, não sendo obtido resultado eficaz. Foi realizado exame clínico geral e coleta de sangue para realização de hemograma (coleta de sangue da veia jugular em tubo contendo EDTA). Na inspeção otológica foi observado que o conduto auditivo esquerdo apresentava um aumento de volume no terço médio e, na palpação do pavilhão auricular, foi observada a presença de uma massa consistente. Essa massa foi mensurada com o auxílio de um paquímetro e tratada com aplicações durante 2 meses com $1 \mathrm{mg} / \mathrm{kg}$ de acetato de metilprednisolona infiltrativo em duas doses com intervalo de 30 dias (Figura 1). Os resultados do tratamento foram avaliados a cada 30 dias, através da mensuração do aumento de volume em sentido longitudinal e transversal, com a utilização de um paquímetro, para acompanhar o progresso do tratamento.

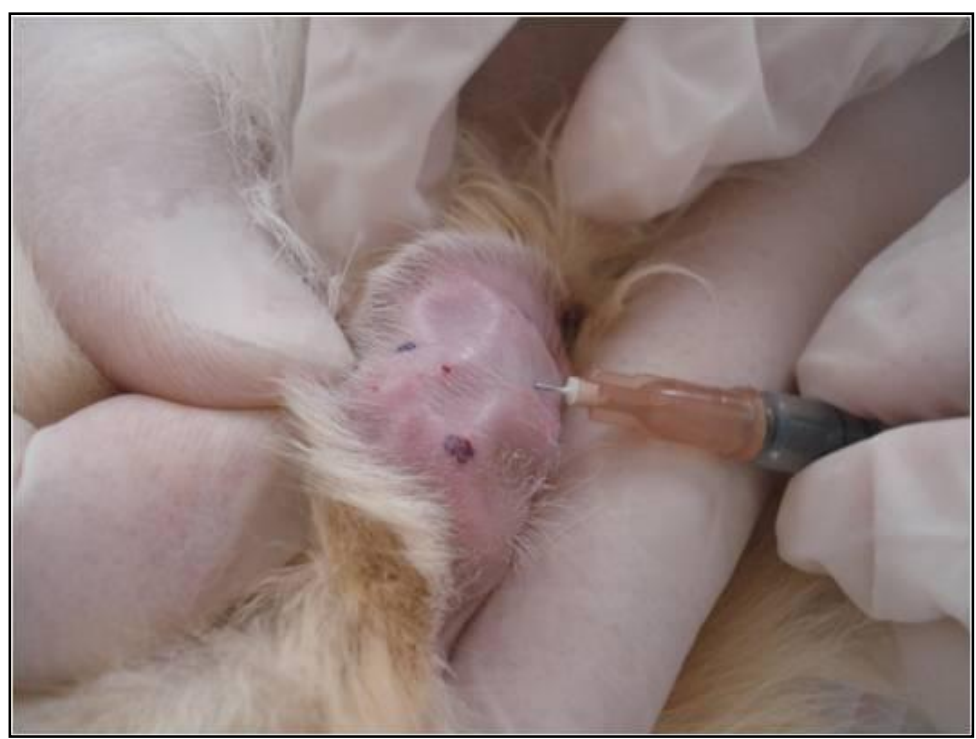

Figura 1- Demonstração da aplicação de $1 \mathrm{mg} / \mathrm{kg}$ de metilprednisolona infiltrativa em um felino, macho, persa de três anos de idade com presença de fibrose em formato retangular no pavilhão auricular esquerdo. 


\section{RESULTADOS E DISCUSSÃO}

O exame clínico geral e hemograma do paciente não apresentaram alterações, porém a inspeção da orelha foi constatada que o aumento de volume era devido a uma área fibrótica em formato retangular na concha acústica esquerda determinando irregularidade anatômica da mesma. A terapêutica de eleição para a correção do otohematoma é a cirúrgica, pois apresenta uma baixa taxa de recidivas e proporciona que o pavilhão auricular externo apresente aparência estética harmônica. No entanto, nas drenagens do pavilhão auricular ocorre uma alta taxa de recidiva além da ocorrência de um padrão estético não harmônico da orelha, sendo essa a possível causa da fibrose do pavilhão auricular do felino (SCHIOCHET et al., 2010).

Na primeira avaliação do pavilhão auricular, a massa fibrótica apresentava uma área retangular de $200 \mathrm{~mm}^{2}$ ao ser mensurada, devido a isso o animal apresentava uma leve inclinação da cabeça para o lado esquerdo em razão do aumento de volume do pavilhão auricular. Na segunda avaliação, 30 dias após a aplicação de metilprednisolona infiltrativa, foi observada, na palpação e inspeção do pavilhão auditivo, uma diminuição da área de fibrose e o paciente apresentava a cabeça ereta. Após a aferição da área com o paquímetro foi constatada uma massa $140 \mathrm{~mm}^{2}$ de fibrose confirmando a diminuição da mesma (Figura 2). Na terceira avaliação, aos 60 dias de tratamento, a medição da área de fibrose apresentou $137 \mathrm{~mm}^{2}$ (Figura 3). Como o paciente apresentava a cabeça ereta e a massa tinha reduzido consideravelmente seu tamanho apresentando assim um aspecto da pina auricular próximo à normalidade, os proprietários optaram por cessar o tratamento, pois estavam satisfeitos com a melhora do gato.

Entre as duas primeiras avaliações houve uma diminuição considerável da massa, pois o glicocorticóide afeta a síntese e maturação do colágeno além de inibir a função do fibroblasto resultando dessa forma na alteração do padrão de uma cicatriz fibrótica. No entanto entre a segunda e terceira, a retração da massa foi pequena, podendo ser justificado possivelmente pelo glicocorticóide não ter agido na região central do tecido fibroso (CAMPAGNOLO et al, 2008; HAMMER et al., 2004). Dessa forma, demonstrando o 
resultado visto nesse trabalho, em que nas primeiras aplicações do tratamento houve uma resposta satisfatória, porém ao final da terapia farmacologia a resolução não foi total.

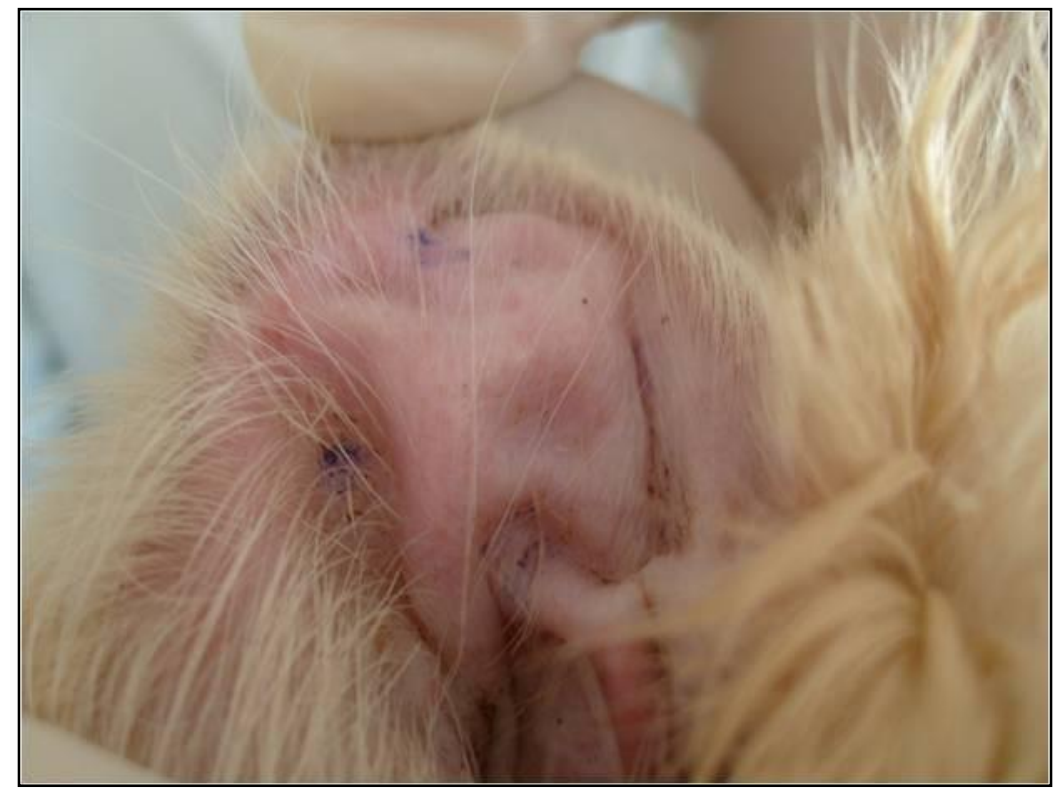

Figura 2- Demonstração da área de fibrose retangular no pavilhão auricular esquerdo em um felino, macho, persa de três anos de idade, após uma aplicação de $1 \mathrm{mg} / \mathrm{kg}$ de metilprednisolona infiltrativa (30 dias de tratamento).

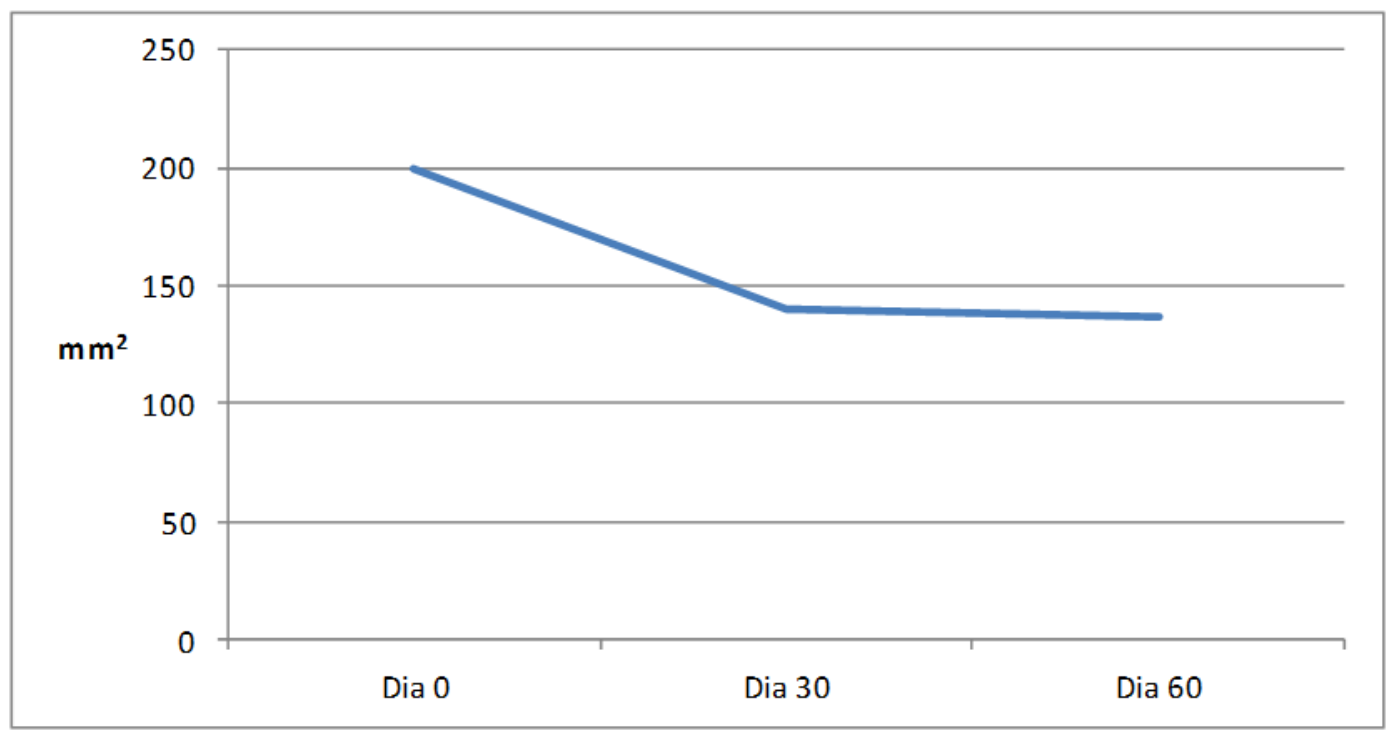

Figura 3- Representação gráfica da diminuição da área de fibrose retangular $\left(\mathrm{mm}^{2}\right)$ no pavilhão auricular esquerdo de um felino, macho, persa de três anos de idade. 


\section{CONCLUSÃO}

Conclui-se através desse relato de caso que a aplicação por dois meses com $1 \mathrm{mg} / \mathrm{kg}$ de acetato de metilprednisolona intralesional em duas doses com intervalos de 30 dias entre as aplicações, apresenta resultado satisfatório na redução da fibrose auricular decorrente do otohematoma.

\section{EAR FIBROSIS DUE TO AURAL HEMATOMA IN A CAT: GLUCOCORTICOID THERAPY - CASE REPORT}

\section{ABSTRACT}

$\mathrm{T}$ he aural hematoma is characterized by the accumulation of blood in the convex side of the ear, which may cause scarring if not treated properly. The aim of this study is to report the case of a cat with the presence of fibrosis in the left pinna, which was treated with infiltrative glucocorticoid. The patient had a history of non-treated aural hematoma and the presence of a fibrous mass in the left ear and a slight tilt to the same side. The fibrosis was treated for two months with $1 \mathrm{mg} / \mathrm{kg}$ of infiltrating methylprednisolone administered with an interval of 30 days. The ear of the cat was evaluated every 30 days by otoscopy and the fibrous mass was measured with a calliper. The fibrous mass inspection showed a gradual reduction of the fibrosis area in each evaluation (200, 140 and $137 \mathrm{~mm}^{2}$ ), and in the second evaluation the patient's head was upright. Thus, it is possible to conclude that the administration of $1 \mathrm{mg} / \mathrm{kg}$ of infiltrating methylprednisolone for two months with an interval of 30 days between the applications, provided satisfactory results for the treatment of aural fibrosis due to an ear hematoma.

Keywords: Corticosteroids. Diseases. Cat. Ear. 


\section{FIBROSIS AURICULAR SECUNDARIA A OTOHEMATOMA EN FELINO: TERAPÉUTICA CON GLUCOCORTICOIDE - INFORME DE CASO}

\section{RESUMEN}

$\mathrm{E}$

I otohematoma se caracteriza por la acumulación de sangre en la superficie convexa de la oreja desarrollando fibrosis cicatricial si no se trata adecuadamente. El objetivo de este trabajo fue relatar el caso de un felino con presencia de fibrosis en el pabellón auricular izquierdo, tratado con acetato de metilprednisolona infiltrativo. El paciente presentaba histórico de otohematoma no tratado quirúrgicamente, aumento de volumen en el pabellón auricular izquierdo y una ligera inclinación de la cabeza hacia el mismo lado. La fibrosis fue tratada durante dos meses con dos dosis de $1 \mathrm{mg} / \mathrm{kg}$ de metilprednisolona infiltrativa en la lesión, con intervalos de 30 días. El conducto auditivo del felino fue evaluado a cada 30 días por otoscopia y medida la masa fibrosa con un calibrador. En la inspección de la masa fibrosa fue verificada reducción gradual del área de la fibrosis en cada evaluación (200, 140 y $137 \mathrm{~mm}^{2}$ ) siendo que en la segunda evaluación el paciente presentaba la cabeza erguida. Concluyendo de esta manera que la aplicación de $1 \mathrm{mg} / \mathrm{kg}$ de metilprednisolona infiltrativa durante 60 días con intervalo de 30 días entre las aplicaciones presenta resultado satisfactorio para el tratamiento de la fibrosis auricular secundaria a un otohematoma.

Palabras clave: Corticoide. Enfermedades. Gato. Oído.

\section{REFERÊNCIAS}

CAMPAGNOLO, A. M.; SENNES, D. H. T. L. U; IMAMURA, R. Injeção de corticosteroide em patologias vocais inflamatórias crônicas, revisão da literatura. Revista Brasileira de Otorrinolaringologia, São Paulo, v. 74, n. 6, p. 926-932, nov./dez. 2008.

COLE, L. K. Otoscopic Evaluation of the Ear Canal. The Veterinary Clinics of North AmericaSmall Animal Practice, Atlanta, v. 34 , n. 2, p. 397-410, mar. 2004.

EVANGELISTA, L. S. M.; CARVALHO, Y. N. T.; BRANCO, M. A. C.; et al. Estudo Retrospectivo do Otohematoma em Cães Atendidos em um Hospital Veterinário Universitário. Acta Veterinaria Brasilica, Mossoró, v. 6, n. 1, p. 48-51, jan./mar. 2012.

HAMMER, S.; SAUER, B.; SPIKA, I.; et al. Glucocorticoids mediate differential anti-apoptotic effects in human fibroblasts and keratinocytes via sphingosine-1-phosphate formation. Journal of Cellular Biochemistry, Nova Jersey, v. 91, n. 4, p. 840-851, mar. 2004. 
HARVEY, R.G.; HARARI, J.; DELAUCHE , A. J. Doenças do Ouvido em Cães e Gatos. Rio de Janeiro: Revinter, 2004. 272p.

LOBELL, R., WEINGARTEN, A., SIMMONS, R. Um novo agente para o tratamento da otite externa canina. A Hora Veterinária, Porto Alegre, v. 88 n. 1, p. 29-33, jan./fev. 1995.

NASCENTE, P. S.; CLEFF, M. B.; ROSA, C. S.; et al. Otite externa em pequenos animais: uma revisão. MEDVEP - Revista Científica de Medicina Veterinária - Pequenos Animais e Animais de Estimação, Curitiba, v. 4, n. 11, p. 52-59, jun. 2006.

SCHIOCHET, F.; TEIXEIRA, E.; RODRIGUES, P. R. C.; et al. Hematoma auricular em gato: relato de caso. Veterinária em Foco- Revista de Medicina Veterinária, Canoas, v. 7, n. 2, p. 198205, jan. /jun. 2010.

SCHOSSLER, J. R.; MÜLLER, D.; PINHEIRO, M. Proposição de técnica para drenagem de otohematoma em cães. Arquivo Ciências Veterinária e Zoologia da Unipar, Umuarama, v. 10, n. 2, p. 117-119, jul./dez. 2007.

Autor para correspondência: Mariana Teixeira Tillmann Doutoranda do Programa de Pós Graduação em Veterinária- UFPEL. ClinPet-Grupo de Ensino, Pesquisa e Extensão em Clínica de Pequenos Animais, Faculdade de Veterinária, Campus Capão do Leão, UFPEL -

Cep:96160-000. mariana.teixeira.tillmann@gmaul.com 